\title{
Underground cosmic-ray experiment EMMA
}

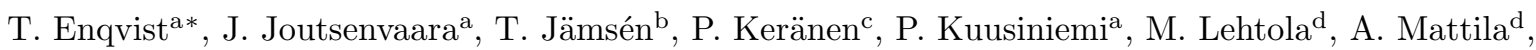
J. Narkilahti ${ }^{d}$, J. Peltoniemi ${ }^{d}$, A. Pennanen ${ }^{\text {a }}$, T. Räihä ${ }^{\mathrm{d}}$, J. Sarkamo ${ }^{\mathrm{d}}$, C. Shen ${ }^{\mathrm{a}}$, W. Trzaska ${ }^{\mathrm{e}}$

I. Usoskin ${ }^{\mathrm{b}}$, M. Vaittinen ${ }^{\mathrm{d}}$, and Z. Zhang ${ }^{\mathrm{a}}$

${ }^{a}$ Centre for Underground Physics at Pyhäsalmi (CUPP), University of Oulu, P.O. Box 22, FI-86801

Pyhäsalmi, Finland

bSodankylä Geophysical Observatory (SGO), University of Oulu, Oulu, Finland

${ }^{\mathrm{c}}$ Radiation and Nuclear Safety Authority - STUK, Helsinki, Finland

${ }^{\mathrm{d}}$ Centre for Underground Physics at Pyhäsalmi (CUPP), University of Oulu, Oulu, Finland

eDepartment of Physics, University of Jyväskylä (JYFL), Jyväskylä, Finland

A new cosmic-ray experiment is under construction in the Pyhäsalmi mine, Finland. It aims to study the chemical composition of cosmic rays at and above the knee region. The array, called EMMA, will cover approximately $150 \mathrm{~m}^{2}$ of detector area at the depth of 85 metres ( $\left.240 \mathrm{mwe}\right)$. It is capable of measuring the multiplicity and the lateral distribution of underground muons, and the arrival direction of the air shower. The full-size array is expected to be ready by the end of 2007. A partial-size array (one third of the full size) is planned to record data already at the first quarter of 2007. The array is also expected to be capable of measuring such high-multiplicity muon bundles as was observed at the cosmic-ray experiments at the LEP detectors.

\section{INTRODUCTION}

Due to a slight change observed in the cosmicray energy spectrum in the energy interval of $10^{15}$ $-10^{16} \mathrm{eV}$, it is believed that the origin, modification in the chemical composition, acceleration mechanism or propagation of cosmic rays (or a combination of these) changes. Up to this energy, the so called knee region, most cosmic rays are supposed to be produced inside the galaxy, and are also confined by the galactic magnetic field.

At these high energies the source cannot be observed directly and the cosmic-ray composition is used as a tool to investigate the origin of cosmic radiation. The direct composition measurements are no longer practical at or above the knee and the method is solely based on the measurement of extensive air showers, i.e. secondary particles created in the atmosphere and detected usually

*Corresponding author.

Email address: timo.enqvist@oulu.fi (T. Enqvist) by large arrays on the ground.

The origin of the knee has been one of the fundamental problems of cosmic-ray physics, and it has been discussed for decades. Several models have been presented predicting different composition at the knee energies, and could only be identified by the experimental evidence on the composition. Some new experimental efforts have been devoted to the study of cosmic rays in recent years. These experiments are based, for example, on multi-parameter measurements of extensive air-showers, on shower maximum measurement by Čerenkov or fluorescence detectors and on underground multimuon measurements (see, for example, Ref. [1] and references therein). Their conclusions, however, have so far been diverse, implying the need for further studies, especially using different approaches. The results are also known to be strongly model dependent.

EMMA (Experiment with MultiMuon Array) uses a different experimental approach. It is not 


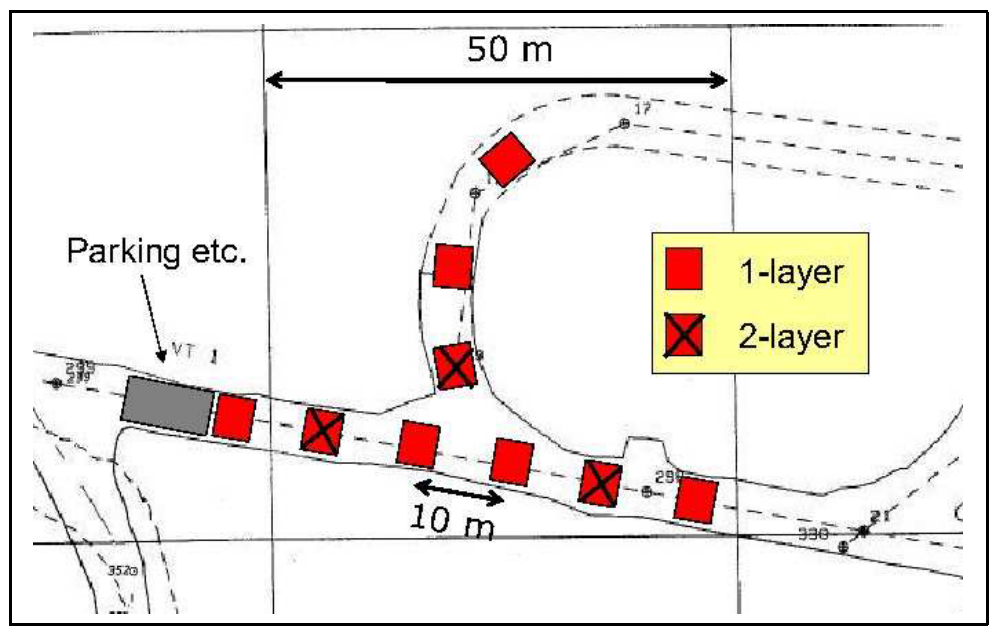

Figure 1. Schematic layout of the EMMA array in the underground site at 85 metres. The array consists of nine units each having an area of about $15 \mathrm{~m}^{2}$. The horizontal and vertical dimensions are approximately 60 and 35 metres, respectively. The three crossed units occupy detectors in two layers for the shower direction measurement. The array will be later on upgraded with plastic scintillation detectors.

the first underground cosmic-ray experiment (see, for example, Refs. [2-6]), but it differs significantly from previous underground experiments with its ability to measure the lateral distribution function of high-energy muons. In EMMA the composition analysis is based on the lateral distribution of high-energy muons and their multiplicity. The muons detected by EMMA are generated in the upper part of the air shower close to the primary interaction.

\section{EXPERIMENTAL DETAILS}

The present experiment will be carried out at the depth of 85 metres in the Pyhäsalmi mine (owned by the Inmet Mining Corporation Ltd., Canada) situated in the central Finland.

The detector array is placed at the depth of 85 metres (corresponding 240 m.w.e) which gives a threshold energy of muons of approximately 50 $\mathrm{GeV}$. The rock overburden filters out all other charged particles of the air shower except the high-energy muons. A schematic layout of the array is shown in Fig. 1, where each of the nine units have an area of approximately $15 \mathrm{~m}^{2}$. The area between the units is close to the unit area.

The EMMA detector array consists of drift chambers (muon barrel, MUB, detectors) previously used in the LEP-DELPHI experiments at CERN [7]. The setup is able to measure the arrival direction of the air shower, the muon multiplicity and their lateral distribution. This is carried out by an array of separate detectors with the total detector area of approximately $150 \mathrm{~m}^{2}$. A part of the array is in two layers with a vertical distance of approximately 2.5 metres between the layers in order to obtain the direction information (see Fig. 1 for details).

Most of the drift chambers have an active volume of $365 \times 20 \times 1.6 \mathrm{~cm}^{3}$. A plank consists of seven chambers (partly overlapped) having an area of approximately $3 \mathrm{~m}^{2}$. The layout shown in Fig. 1 requires 60 planks. The drift chambers operate in the proportional mode, with Ar: $\mathrm{CO}_{2}$ (92:8) nonflammable gas mixture. Due to the safety issues, the gas mixture does not contain formerly used $\mathrm{CH}_{4}$. Each drift chamber can provide up to three signals, one anode signal and 

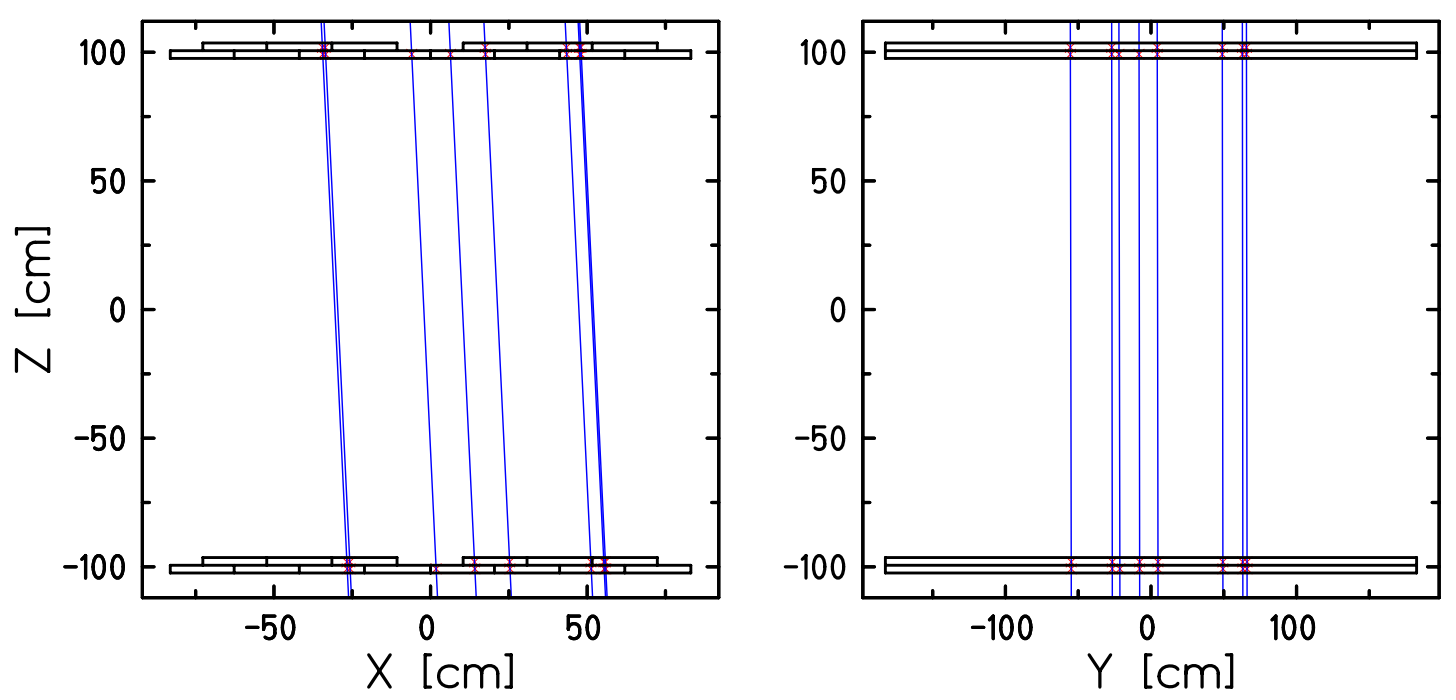

Figure 2. Eight muons hitting two planes of detectors with randomly chosen shower direction and muon hit positions. The lines connecting the hits in the upper and lower planes result from a tracking code. In this example, there are only two planks per plane.

two delay-line signals (near and far), which can be used for position information of particles passing through the chambers. The measured position resolution of the chambers in our setup is about $1 \mathrm{~cm}$ and $3 \mathrm{~cm}$ along the drift direction and along the drift line, respectively.

The above values for the position resolutions were measured with a slow trigger (or self trigger). It means that the trigger signal was taken from a drift chamber. The experiment will be started using a slow trigger. It will be upgraded later by a fast (or external) trigger where the start signal is taken from plastic scintillation detectors. With the fast trigger the position resolutions would be nearly the same as in the DELPHI experiments.

The acceptance, assuming an area where the shower axis can be determined with an accuracy better than 6 metres, was estimated to be approximately $300 \mathrm{~m}^{2} \cdot \mathrm{sr}$ for $4-\mathrm{PeV}$ proton-initiated shower.

Recycling the old muon barrel detectors pro- vides a possibility to built the array at low costs. Furthermore, the use of existing caverns in the mine reduces the costs. A part of data-acquisition electronics is build by ourselves and the rest bought as new.

\section{MUON TRACKING}

An important part of the data analysis software is the muon tracking routine. As it is obvious that an observed shape of the shower partially depends on the angles it hits the detectors, the shower can only be reconstructed by determining the angles of individual muons. In EMMA the shower reconstruction will be carried out by tracking muons as they hit simultaneously to the three two-layer units.

The simulated performance of the muon tracking routine is illustrated in Fig. 2 using an example where eight simultaneous muons penetrate two detector planes with a randomly chosen shower angle. The tracking code employs an automatic routine which connects the pairs in two 


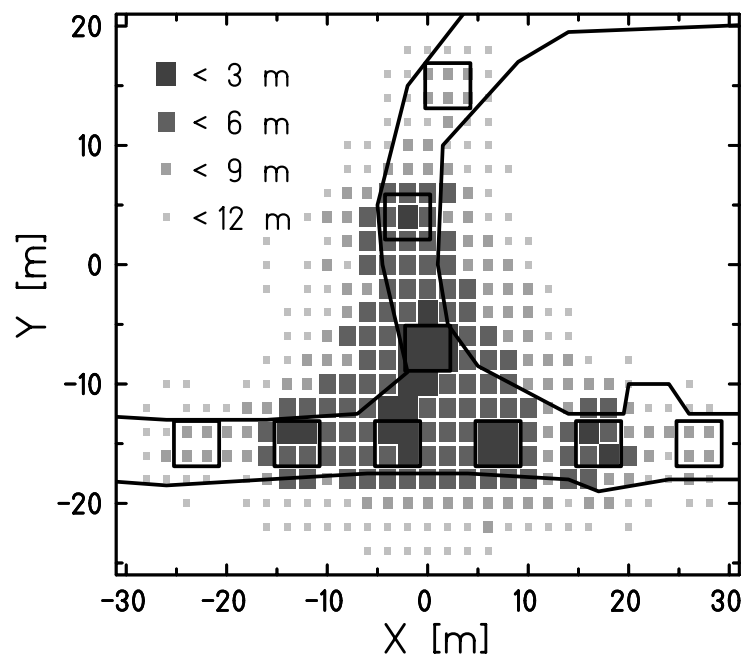

Figure 3. The accuracy of the reconstructed shower axis is shown as a cluster plot for a $4-\mathrm{PeV}$ proton shower. The symbols represent the average shower axis uncertainty. The area where the shower axis can be determined with an average accuracy better than 6 metres is approximately $300 \mathrm{~m}^{2}$. Each cluster contains 300 showers.

planes and rejects pairs too far from the average angle.

\section{LOCATING SHOWER AXIS}

The reconstruction accuracy of the shower axis depends slightly on the hit position. With the current detector layout (see Fig. 1) the average accuracy of better than 6 metres can be achieved in a large collection area. This is illustrated in Fig. 3, where 300 proton-initiated showers at 4 $\mathrm{PeV}$ have been dropped into each cluster. In this example, the collection area is approximately 300 $\mathrm{m}^{2}$ in which the shower-axis reconstruction is on average better than 6 metres.

The lateral distribution function of high-energy muons was parametrised as

$\rho(r)=\frac{N_{\mu}}{2 \pi \cdot 0.11 \cdot R_{0}^{2}} \cdot\left(\frac{r}{R_{0}}\right)^{-0.4} \cdot\left(1+\frac{r}{R_{0}}\right)^{-5}$ using CORSIKA (QGSJET01c) [8,9] simulations. In Eq. (1), $\mathrm{r}$ is the distance from the shower axis, $\mathrm{N}_{\mu}$ is the total number of muons, and $\mathrm{R}_{0}$ is related to the gradient of the lateral distribution function. At the knee energies various air-shower models result in approximately similar shapes for the lateral distribution function.

In order to locate the shower axis, a twodimensional fit routine employing Eq. (1) was developed. The density distribution of the hit coordinates of muons (bin size $1 \times 1 \mathrm{~m}^{2}$ ) is used as an input for the routine (together with the assumption of the shape). The output values of the fitting routine are the shower location, total number of muons in the shower (above the threshold energy), and parameter $R_{0}$.

\section{EXTRACTING COMPOSITION}

The idea of extracting primary cosmic-ray mass composition is based on the fact that, according to cosmic-ray air-shower models (e.g. CORSIKA \& QGSJET $[8,9])$ the lateral distribution, or its gradient, of high-energy muons is sensitive to primary cosmic-ray particles and their energies (see Fig. 4). The number of muons at the core can be used as an indicator for the primary energy. The energy resolution of EMMA according to this preliminary analysis is somewhat moderate.

As no experimental results have been obtained yet, expected results based on simulations are intruduced in this section to present some of the performance of the EMMA array. The air-shower simulations and particularly the data analysis methods are still preliminary but they already indicate good capabilities for the EMMA array.

The composition reconstruction simulations have been performed with a realistic energy spectrum of primary cosmic rays and various protoniron fractions, i.e. a two-component model was assumed for the composition. The energy interval was between 0.8 to $20 \mathrm{PeV}$ and the spectral index of the power law of -2.7 was used. The shower axes were distributed uniformly in and around the detector array (in an area of $160 \times 160 \mathrm{~m}^{2}$ ). The simulated sample consisted of $2 \times 10^{6}$ showers. The total data set then corresponds roughly to one year $\times$ steradian of data. 


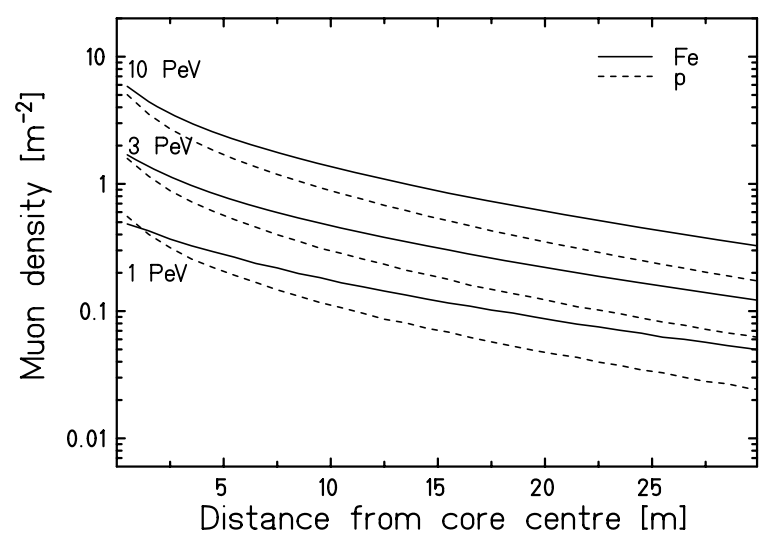

Figure 4. Lateral distribution of muons for proton- and iron-initiated air showers with three different initial energies. The energy of muons is higher than $45 \mathrm{GeV}$.

The data for the composition reconstruction was selected by applying two cuts to the total data set. First, there needed to be more than ten hits in at least one detector unit. Second, the reconstructed shower axis was within a triangular area surrounding the three centermost units. By fitting Eq. (1) resulted parameter $\mathrm{R}_{0}$ and the value of the lateral distribution one metre from the core $(\rho(1))$. All showers were fitted and a two-dimensional distribution of $\mathrm{N}\left(\rho(1), \mathrm{R}_{0}\right)$ was created. The response functions for monoenergetic showers $\mathrm{f}_{\mathrm{i}}^{\mathrm{p}}\left(\rho(1), \mathrm{R}_{0}\right)$ and $\mathrm{f}_{\mathrm{i}}^{\mathrm{Fe}}\left(\rho(1), \mathrm{R}_{0}\right)$ were created similarly.

To reconstruct the primary composition, a maximum likelihood analysis was done by minimising the error of

$\mathrm{N}\left(\rho(1), \mathrm{R}_{0}\right)=\sum_{\mathrm{i}}\left(\mathrm{P}_{\mathrm{i}} \times \mathrm{f}_{\mathrm{i}}^{\mathrm{p}}+\mathrm{Fe}_{\mathrm{i}} \times \mathrm{f}_{\mathrm{i}}^{\mathrm{Fe}}\right)$,

where the variables $\mathrm{P}_{i}$ and $\mathrm{Fe}_{\mathrm{i}}$ describe the amount of each individual component in the data in the energy bin i. The outcome of the method is illustrated in Fig. 5 where the separation of light and heavy cosmic-ray primary component is shown.

\section{HIGH MUON MULTIPLICITIES}

In the cosmic-ray experiments at LEP (DELPHI, CosmoALEPH, and L3+C) $[10,4,11]$ events with high muon multiplicities were observed. While the small and medium muon bundles seem to fit into the model predictions, the production of the high multiplicities (above 80 or so) is not yet clear. They were short cosmic-ray runs, effective running times of only about a month each.

The LEP experiments were not able to get information on the lateral distribution of highenergy muons, and thus not directly on the primary particle producing the muon bundle.

EMMA is also able to study the highmultiplicity events, already with a partial-size array. It is expected that EMMA can make a significant contribution to clear out the reason for the highest multiplicities. First, EMMA can measure the lateral distribution and thus obtain good information on the primary particle. In addition, the advantage of EMMA is that this phenomena can now be studied for several years.

\section{SCHEDULE}

The array is under construction. It is expected that measurements could start with a partial-size array (one-third of the array) at the first quarter of 2007. These would be mainly test measurements since the partial-size array is too small to extract information on the chemical composition of cosmic rays. The construction work of the full array is expected to be ready by the end of 2007 .

However, the partial-size array is large enough to observe the possible high muon-multiplicity events. As durations for these measurements at CERN were rather short, we may measure such events with good statistics already with the partial-size array together with the construction of the rest of the array during 2007.

\section{SUMMARY}

A new underground cosmic-ray experiment EMMA is under construction and it is expected to start recording data in the full scale by the end of 2007. With a partial-size array the data recording can be started earlier. The analysis 

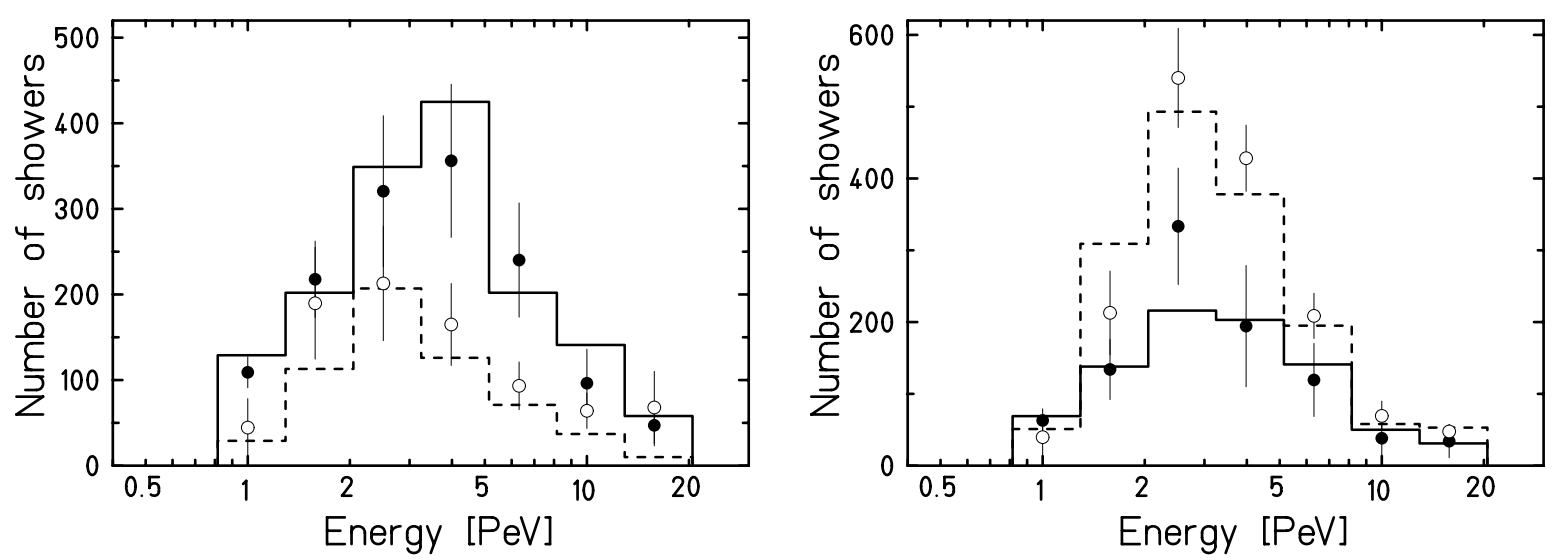

Figure 5. Reconstructed compositions for two different initial conditions: left-hand panel: $50 \%$ proton and $50 \%$ iron, right-hand panel: $80 \%$ proton and $20 \%$ iron. Full histogram shows simulated proton composition and dashed histogram simulated iron composition. Full and open symbols show the reconstructed proton and iron showers, respectively.

of simulated air showers shows that the primary cosmic-ray composition could be resolved (with a two-component model) at and above the knee energies. Due to the new method used, the EMMA experiment could provide comprehensive (and perhaps new) information on the composition of the cosmic rays at the knee region within the next few years. It is also expected that the anomaly of high-multiplicity muon bundles observed at CERN can be studied systematically with EMMA.

\section{ACKNOWLEDGEMENTS}

The support from the Magnus Ehrnrooth Foundation, the Jenny and Antti Wihuri Foundation, the Finnish Academy of Science and Letters (Väisälä Foundation), and the Finnish Cultural Foundation is acknowledged. The work is funded by the European Union Regional Development Fund and it is also supported by the Academy of Finland (projects 108991, 7108875 and 7106570).

\section{REFERENCES}

1. Jörg R. Hörandel, astro-ph/0508014 (2005).
2. D. Cebula et al., The Astrophysical Journal 358 (1990) 637.

3. S.M. Kasahara et al., Physical Review D55 (1997) 5282.

4. V. Avati et al., Astroparticle Physics 19 (2003) 513.

5. C. Grupen et al., Nuclear Instruments and Methods A510 (2003) 190.

6. EAS-TOP Collaboration \& MACRO Collaboration (M. Aglietta et al.), Astroparticle Physics 20 (2004) 641.

7. DELPHI Collaboration, Nuclear Instruments and Methods A303 (1991) 233.

8. D. Heck et al., Report Forschungzentrum Karlsruhe 6019 (1998).

9. N.N. Kalmykov et al., Nuclear Physics B (Proc. Suppl.) 52B (1997) 17.

10. Petr Travnicek, Detection of high-energy muons in cosmic-ray showers, Charles University, Prague, 2003, PhD. Thesis.

11. H.G.S. Wilkens, Experimental study of highenergy muons from extensive air showers in the energy range $100 \mathrm{TeV}$ to $10 \mathrm{PeV}$, University of Nijmegen, 2003, PhD. Thesis. 